UDK 528.83

\title{
A GEOSTATISTICAL APPROACH FOR PREDICTING THE TOP PRODUCING FORMATION IN OIL FIELDS
}

\author{
Mohammad Abdideh ${ }^{1}$, Majed Abyat ${ }^{2}$ \\ Department of Petroleum Engineering, Omidiyeh Branch, Islamic Azad University, Omidiyeh, Iran \\ E-mail: ${ }^{1}$ abdideh@iauo.ac.ir (corresponding author)
}

Received 13 July 2012; accepted 21 September 2012

\begin{abstract}
Drilling engineer's understanding of the subsurface conditions of oil-rich regions in Iran is based on experience and through quantitative assessment of these valuable data. The usage of geostatistical methods converts the qualitative experience to quantitative and provides the way for better result.

In Iranian oil fields, during the drilling operation Asmari formation is of great importance because most of oil reservoirs are located in this formation. In this study, 53 wells in the field were randomly selected, and after studying and reviewing the drilling and geological reports, 40 wells were selected for inclusion in the model. After analysis of the information, a model which predicts the top of Producing Formation accurately was presented.
\end{abstract}

Keywords: top of formation, geostatistical modelling, variogram, kriging.

\section{Introduction}

In the presence of limited information, any reservoir description involves the use of statistics. Even in conventional reservoir simulations, different interpolations schemes are used to estimate reservoir properties at inter-well locations.

"Geostatistical" models provide interesting solutions to the challenges:

1. The construction of 3-D geologically-realistic representations of heterogeneity.

2. The quantification of uncertainty through the generation of not one but a variety of possible models or "realisations".

Geostatistical procedures are versatile enough to be used for several purposes related to reservoir description. Geostatistics can be used to interpolate and extrapolate the values of reservoir variables at unsampled location and also can provide the quantitative relationship describing the spatial variability of a reservoir property. This includes the distance over which a given variable is related to as well as how that variable is spatially related to other variables.

In any estimation process (in addition to its value), it is desirable to know the relative uncertainties in the estimation.

Geostatistics can provide different ways of defining uncertainties in estimated values at unsampled locations. These uncertainty estimates can be useful in risk analysis.
Application of geostatistics is a three-step procedure:

1. Assumption stationary.

2. Spatial Modelling of sample data.

3. Estimation of a variable value at unsampled locations.

Geostatistical methods are used to describe heterogeneity in reservoirs which scales are smaller than the distance between the wells (Dimitrakopoulos, Desbarats 1997). At present, geostatistical method is a powerful tool in modelling. Generally, the geostatistics is the study of the phenomena change in space or time. Essential components in geostatistics are:

1) Evaluation of the data normal distribution (Normalization Analysis).

2) Variogram Analysis.

3) Kriging.

4) Stochastic Simulation.

Variables used in geostatistics are selected randomly. A random variable is a variable in which any value in its range has a certain probability to occur.

In other words, any value of it has a certain likelihood of occurring (David, Blais 1977).

In geostatistics, variables which demonstrate spatial structure are studied. According to the description above, it is evident that in geostatistics, with the use of data quantity in a known coordinates, the coordinates of that same quantity can be estimated in a point with other known coordinates within the domain where spatial structure is dominant (Ortiz, Deutsch 2002). 


\section{Frequency distribution}

The frequency distribution method is one of the simplest ways to analysis sample data. It summarizes the data in a more compact form than original sample observations. To construct a frequency distribution, the range of the data is divided into intervals called class intervals. It is common practice to use class sizes of equal width, but this is not necessary. The number of measurement falls within a particular class, i.e. is called frequency (Webster, Oliver 2007).

\section{Variogram analysis}

The variogram is the most commonly used geostatistical technique for describing the spatial relationship. Mathematically, it is defined as:

$$
\gamma(h)=\frac{1}{2} V(x(\bar{u})-x(\bar{u}-h)) .
$$

It is half of a variance of the difference between the two values located distance a part because of our assumption for the geosciences data, the difference between the two values increases as the distance increases (the relationship gets weaker). The variogram characterizes the spatial continuity or roughness of a data set. Ordinary one-dimensional statistics for two data sets may be nearly identical, but the spatial continuity may be quite different. Variogram analysis consists of the experimental variogram calculated from the data and the variogram model fitted to the data. The experimental variogram is calculated by averaging one-half of the difference squared of the $\mathrm{z}$-values over all pairs of observations with the specified separation distance and direction. It is plotted as a two-dimensional graph. The variogram model is chosen from a set of mathematical functions that describe spatial relationships. The appropriate model is chosen by matching the shape of the curve of the experimental variogram to the shape of the curve of the mathematical function.

Although the variogram is a fairly simple function, its interpretation is not straightforward.

Variogram analysis is an important part of geostatistical modelling. Usually, the variogram model begins from a nonzero value and increases up to a range called effective range (a), and eventually reaches the constant value called sill. The effective range is the range in which the data spatial structure is relevant, and outside the range, the data effect is independent from each other (Corstange et al. 2008).

Theoretically, the value of variogram for $h=0$ should tend to its minimum value of zero, but in practice, true variograms which are as a result of experience, do not usually follow this condition. The value of variogram for $h=0$ is called Nugget effect. As increased, variogram begins from low values and moves towards a constant limit, so some variograms reach a relatively constant value which after that whatever distance increased the amount of viogram does not change significantly. This relatively constant value which change happens accidentally is called sill.

In geostatistics, variograms which reach a constant sill are more important and appropriate for estimation.
However, the experimental variograms are not sufficient for variogram analysis and appropriate theoretical models should be fitted on it. There are several theoretical models for fitting to experimental variograms (Christakos 2001). The Gaussian or hyperbolic isotropic model assumes a gradual rise for the $y$-intercept. The formula used for this model is:

$$
\gamma(h)=C_{0}+C\left[1-\exp \left(\frac{h^{2}}{A_{0}^{2}}\right)\right],
$$

where $\gamma(h)=$ semivariance for interval distance class $h, h=$ lag interval, $\mathrm{C}_{0}=$ nugget variance $\geq 0, \mathrm{C}=$ structural variance $\geq \mathrm{C}_{0}$, and $A_{0}=$ range parameter. In the case of the Gaussian model, the effective range $\mathrm{A}=3^{0.5} \mathrm{~A}_{0}$, which is the distance at which the sill $\left(\mathrm{C}+\mathrm{C}_{0}\right)$ is within $5 \%$ of the asymptote and the sill never meets the asymptote in the Gaussian models (Hansen et al. 2006).

\section{Kriging}

There are several methods for estimation. In a general classification, they can be divided to geostatistical and classical methods. Classical methods use classical statistics for estimation, while in geostatistical methods the estimation is based on spatial structure in the environment (Haining 2003).

In general, geostatistical estimation is a process in which the value of a quantity in points with known coordinates can be determined using the value of the same quantity at different points with known coordinates. Kriging is an estimation method based on a weighted moving average, and it can be regarded as the Best Linear Unbiased Estimator (BLUE). The most important feature of kriging is error estimation, which the relevant associated error can be calculated (Deutsch 2002). Therefore, for each estimated value, the relevant confidence range can be calculated.

As already mentioned, kriging is a weighted moving average. This estimator is defined as follows:

$$
Z_{v}^{*}=\sum_{i=1}^{n} \lambda_{i} z_{v_{i}}
$$

In the above equation, $Z_{v}{ }^{*}$ is estimation assay, $\lambda_{i}$ is weight or importance of the quantity dependent on the ith sample and $z_{v_{i}}$ is the assay of ith sample. This type of kriging is called linear kriging as it is a linear combination of $\mathrm{n}$ data. The condition of using this estimator is that the variable $\mathrm{Z}$ should have normal distribution. If the variable is not normally distributed, non-linear kriging should be used, or firstly a transformation should be found that transforms the distribution of the desired variable to normal, and then linear kriging should be performed on transformed data.

Kriging compared to other estimation methods has many advantages, some of them include:

In the kriging method, each known sample in estimation of the unknown point is completely dependent upon the spatial structure of the related environment, while in other methods the weights are only dependent on a geometrical characteristic such as distance, and do not react/respond to the changes in spatial structure 
As spatial structure is weakened (increased Nugget effect), the role of the sample position decreases, so that in the case of total Nugget effect, the weights of all the samples will be equal (Gotway, Young 2002).

The increasing effective range, causes the weight of known samples which are far from block or the desired unknown point to be overestimated.

Some of the Kirging characteristics are:

1) Kriging accompanied by any estimation reduces its error, and not only the average error can be calculated but also the error distribution (estimation variance) can be determined in the whole area under study.

2) If the quantitative value is estimated in sampling points, the estimated value should be equal to the measured value, and the estimation variance should fall to zero.

3) Kriging is a function of variogram characteristics of estimated blocks and the geometry of blocks used for estimation, but it does not function as a true value of data.

4) Kriging causes softening of changes (reduced vibrations), i.e. the assay distribution of the estimated blocks relative to true assay of blocks has little changes.

\subsection{Kinds of Kriging according to basic volume}

Point kriging: If the measured value and the values estimated by kriging are attributed to the point, kriging is known as point kriging. In general, point sampling is not possible, and even very small samples have a volume. But if the sample size is small compared with the effective range of variogram, it can be assumed defective by approximation. In this type of kriging, the point variogramis used. This method is most widely used in mapping. Thus, based on the points on sampling grid, first a regular grid of points is defined, known coordinates on the map are estimated by point kriging, and then their contour lines are plotted using a selected algorithm (Kelsall, Wakefield 2002).

Block kriging: In estimating reserves of a deposit, the aim is determining the volume of mineral with a certain assay. For this purpose, the desired parameter can be divided to blocks with known centre coordinates, and then their average assayis estimated by block kriging method.

\subsection{The role of searching radius in estimation by kriging}

If the distance increases, the spatial structure weakens and often disappears.

Thus, the points lying beyond a certain distance (effect radius) from the estimation point have virtually no effect on the estimated point, and there is no need to be involved in the process of this point estimation. The maximum distance, the points which participate in the estimation, is called searching radius. Searching radius can be obtained by variogram. This radius is usually considered equal to or slightly smaller (two thirds) than the effective radius. Searching radius causes participation of fewer points in estimation, but increases the accuracy. Besides determining the searching radius, limitation of the maximum number of points participating in the estimates is considered. Such limitation helps to reduce calculation load and consequently speeds up results.

\section{The studied field}

This oil fields located in the northeast of Ahwaz city, the south-western Iran. In general, these fields are located in the eastern side of the Great Basin of Dezfoul Embayment. The dimensions of the field are approximately 65 kilometres of length and of 7 kilometres width, the distance between the reservoir crest and the deepest water and oil interface reservoir in Asmari Formation is about $2000 \mathrm{~m}$.

\section{Model design}

In this study, for designing this model, 53 wells in the field were randomly selected, and after studying and reviewing the drilling and geological reports, 40 wells were selected for inclusion in the model. Necessary information about these wells, including their location and also the entry point to Asmari Formation were digitally extracted.

In the first step, after entering the data, position of the wells in the field was determined (Fig. 1).

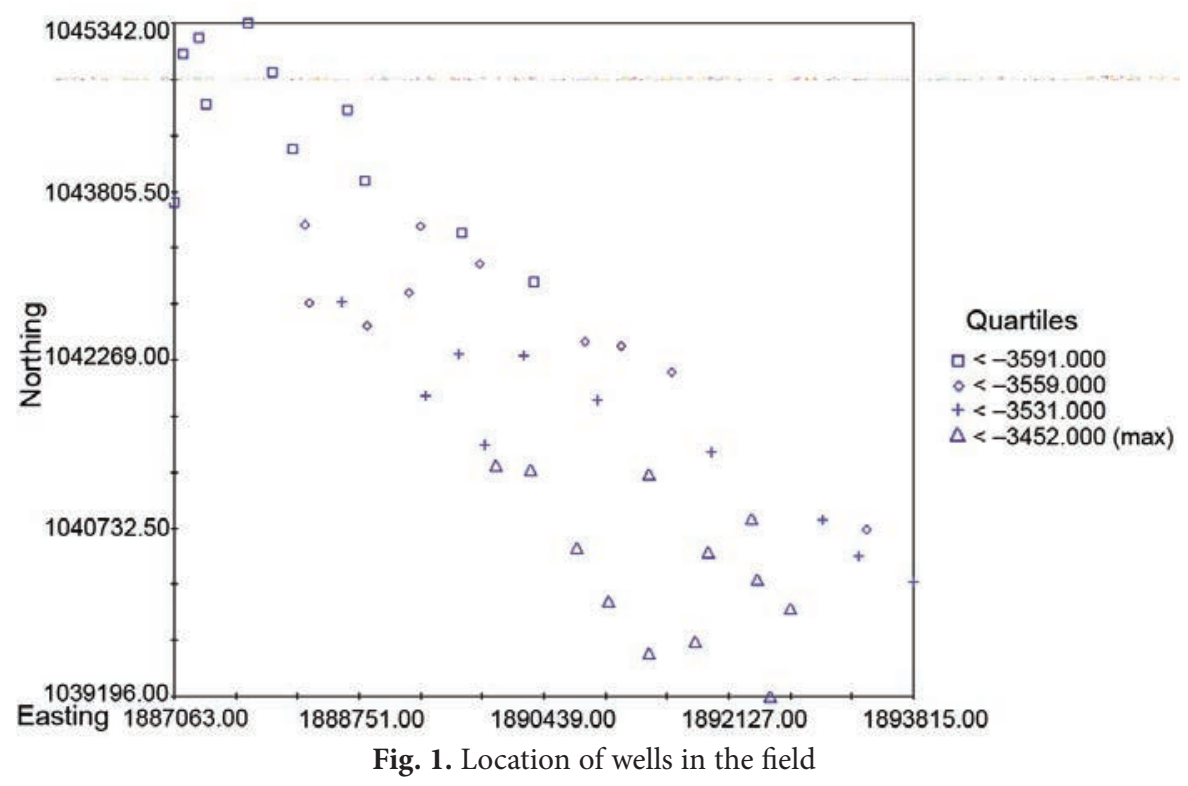


Data analysis, by drawing histograms and cumulative curves, is aimed to determine the type of data distribution (Figs 2, 3). After drawing data histogram, statistical parameters of the data analysis showed a normal distribution (Table 1).

The next step is evaluation of variogram. In this study, after fitting different models and comparing the results (Table 2), the model which selected for fitting was the Gaussian model (Fig. 4). To observe the semi variance variations in different directions, anisotropic variogram surface was plotted. It helps us to determine the main axis of anisotropy in variogram model (Fig. 5).
Table 1. Comparison of statistical parameters before and after normalization of data

\begin{tabular}{l|c}
\hline \multicolumn{1}{c|}{ Statistic Parameter } & Values \\
\hline Mean & -3573.011 \\
\hline Std Deviation & 78.681 \\
\hline Sample Variance & 6190.721 \\
\hline Minimum & -3791 \\
\hline Maximum & -3452 \\
\hline Skewness & -0.87 \\
\hline Kortosis & 0.34 \\
\hline
\end{tabular}

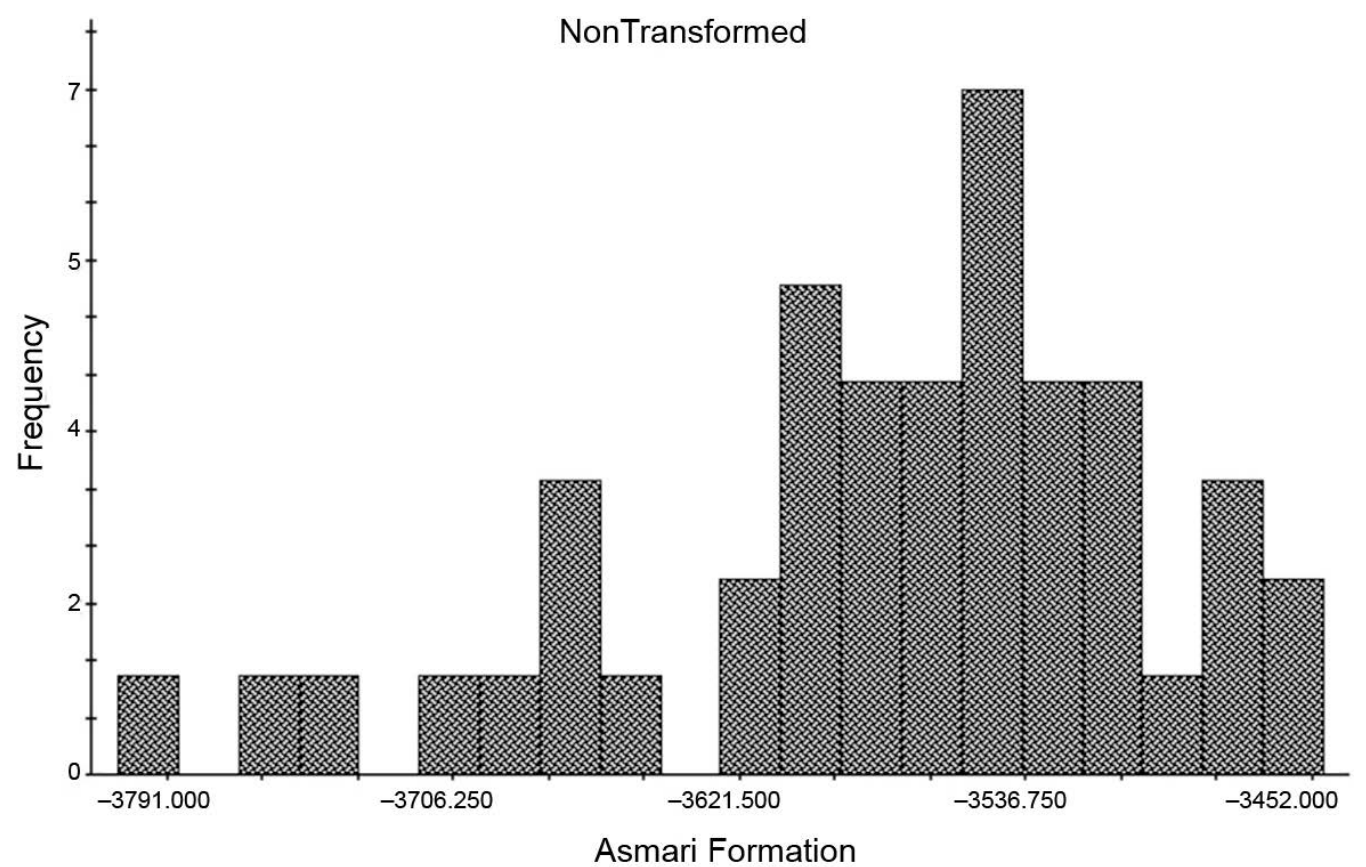

Fig. 2. Frequency distribution of data

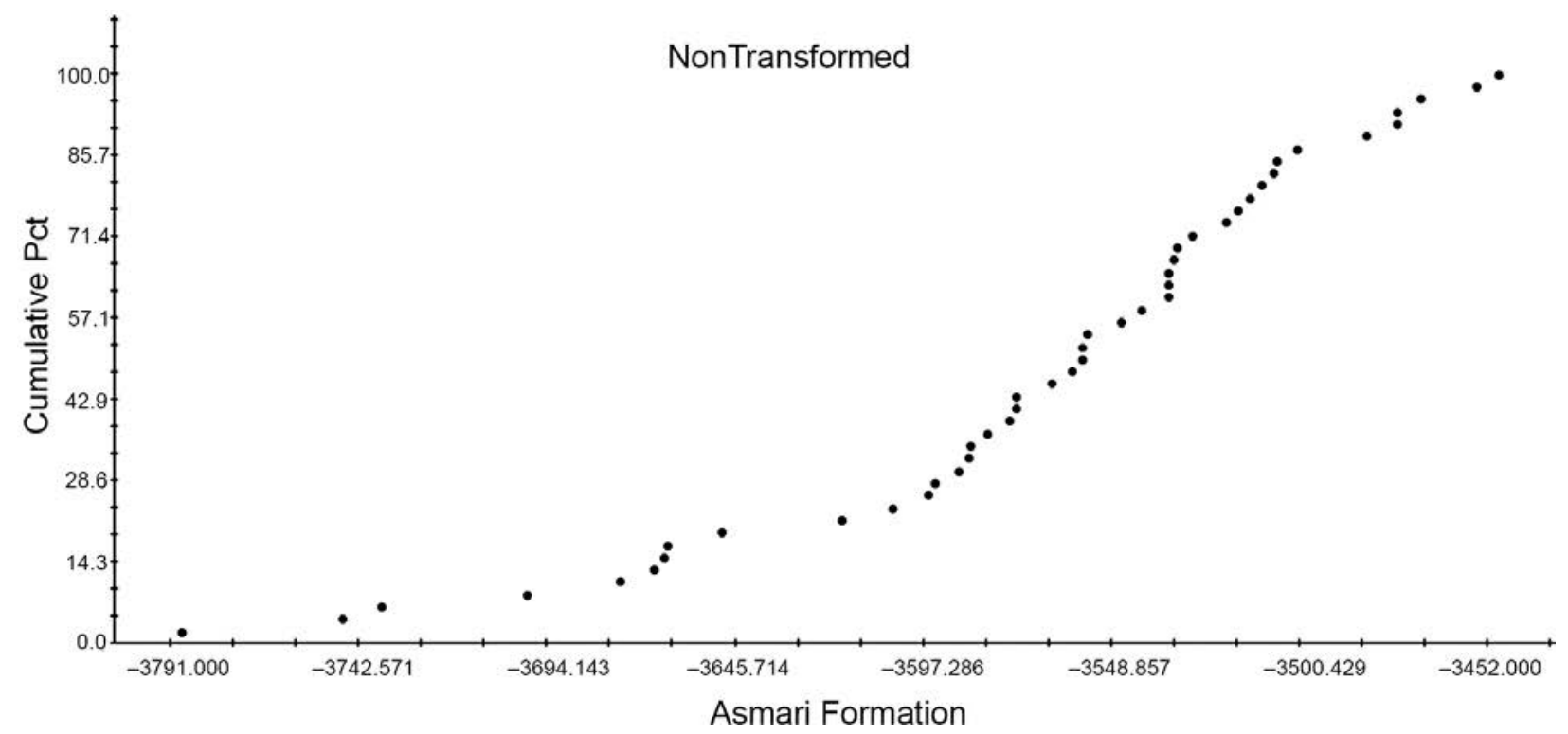

Fig. 3. Cumulative frequency curve of data 
Table 2. Comparison of results from different model

\begin{tabular}{|c|c|c|c|c|c|c|}
\hline \multirow{2}{*}{ Model } & \multirow{2}{*}{$\begin{array}{c}\text { Nugget } \\
\text { (C0) }\end{array}$} & \multirow{2}{*}{$\begin{array}{c}\text { Sill } \\
(\mathrm{C} 0+\mathrm{C})\end{array}$} & \multicolumn{2}{|c|}{ Range Parameter } & \multirow{2}{*}{$\begin{array}{c}\text { Proportion } \\
\mathrm{c} /\left(\mathrm{c}_{0}+\mathrm{c}\right)\end{array}$} & \multirow{2}{*}{$\mathrm{r}^{2}$} \\
\hline & & & Major (A1) & Minor (A2) & & \\
\hline Spherical & 0.01000 & 4.02900 & 24660 & 24660 & 0.998 & 0.860 \\
\hline Exponential & 0.01000 & 4.02900 & 13940 & 41820 & 0.998 & 0.819 \\
\hline Linear & 0.01000 & 3.58200 & 14810 & 14810 & 0.997 & 0.872 \\
\hline Linear to all & 0.01000 & 3.58200 & 14810 & 14810 & 0.997 & 0.872 \\
\hline Gaussian & 0.01000 & 4.02900 & 9180 & 15900 & 0.998 & 0.926 \\
\hline
\end{tabular}

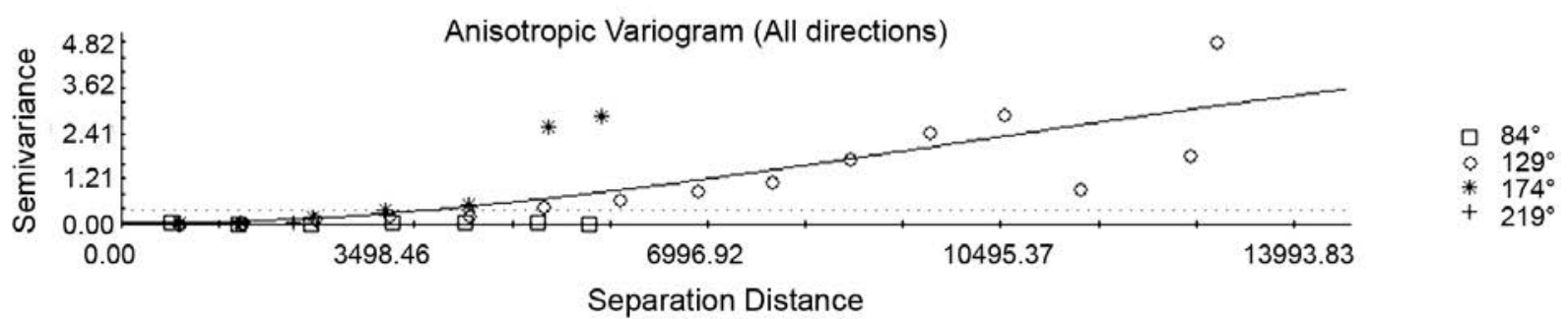

Gaussian model $(C O=0.0240 ; C 0+C=5.3859, A 1=14040.00 ;$ $A 2=14040.00 ; 12=0.524$, RSS $=16.8$ )

Fig. 4. Gaussian model fitted

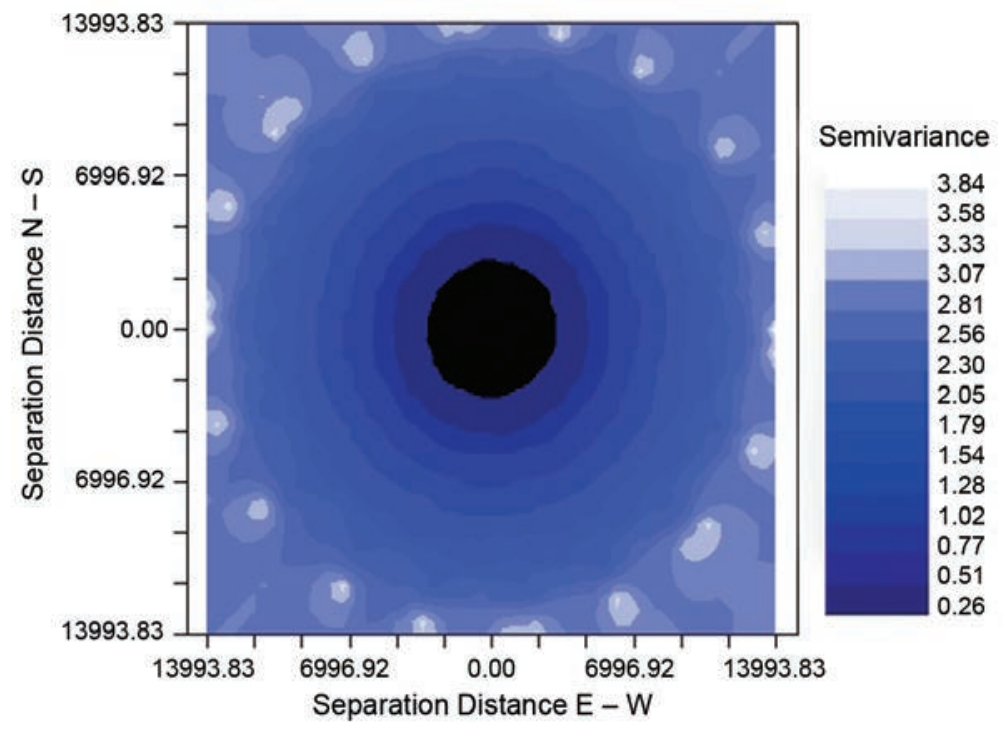

Fig. 5. The model of variogram surface

In this stage, after identifying the structures dominating the estimated space, the proper estimator should be selected. One of the most famous geostatistical estimators is Kriging, which is an unbiased estimator. Estimation can be done using kriging, and estimation error can be introduced using estimation and dispersion variance tools. One of the effective parameter in model design with Kriging is searching radius. In this model, the searching radius is considered equal to effective range.
As already mentioned, kriging has many different types, and in this study the block kriging $(2 \times 2)$ has been used for a model design.

Cross validation is a method for controlling the error accuracy of estimation. In this method, the true value of data and the estimated value are placed close to each other on the same diagram, and the estimation error value is obtained by comparison of these two values (Fig. 6). After complete estimation, a model of formation top is presented (Figs 7, 8). 


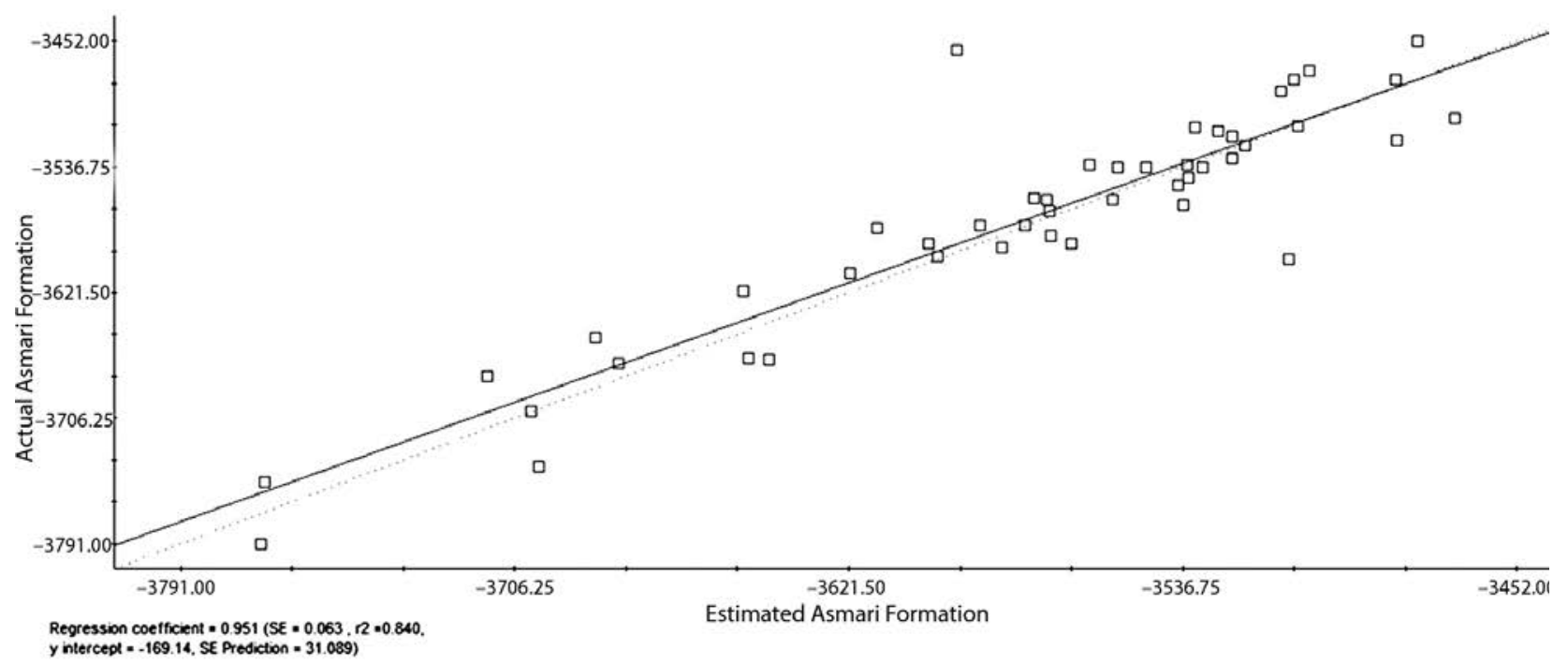

Fig. 6. Cross Validation Diagram for determining Error Estimation

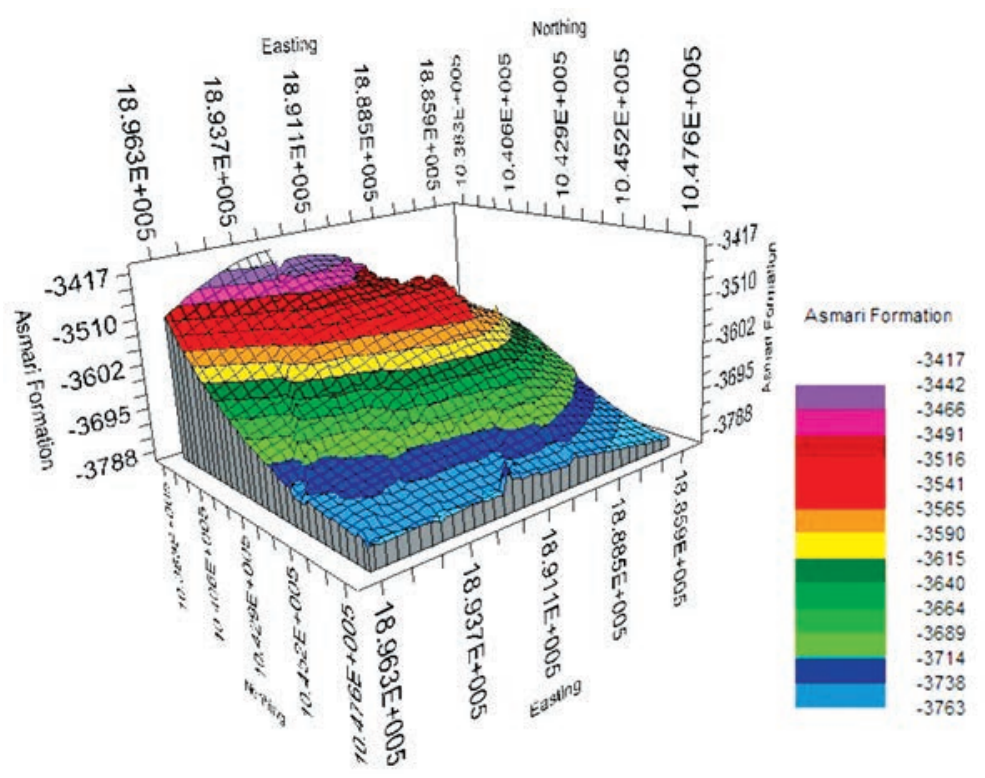

Fig. 7. Final model of the top of Asmari Formation (left view)

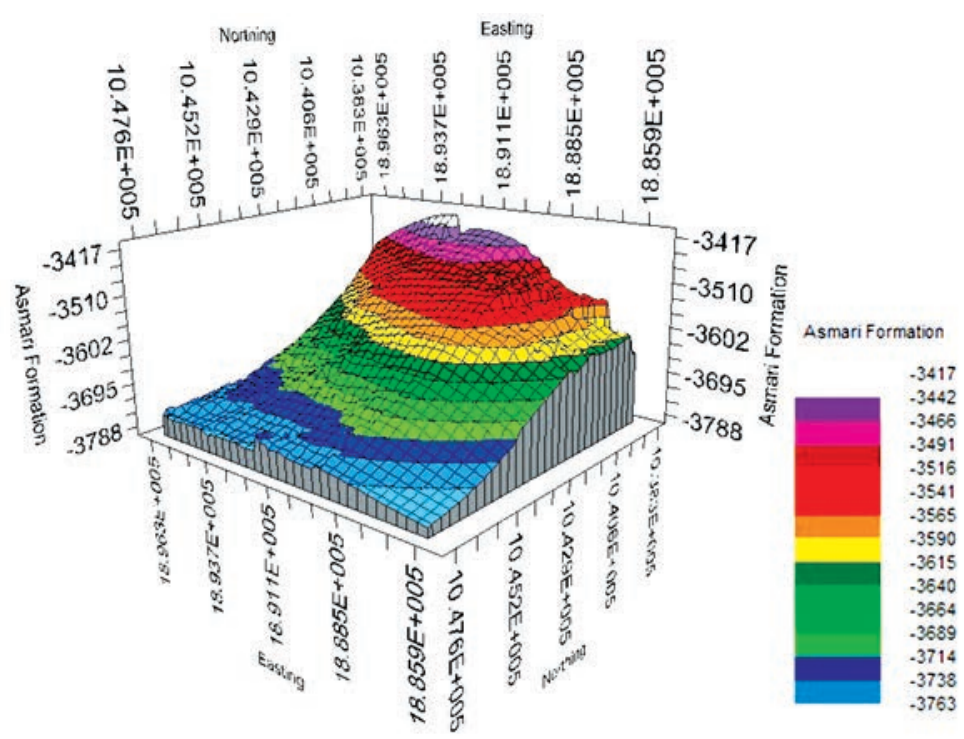

Fig. 8. Final model of the top of Asmari Formation (right view) 


\section{Conclusion}

Top Asmari Formation is one of the most important formations for geologists and drillers in drilling oil wells. It is important because:

1. Most of these oil reservoirs in this formation are located in the southern regions.

2. In this formation losing of drilling mud is relatively heavy.

3. Determination of top level of this formation with purpose of designing casing.

Consequently, predicting the top of this formation is very important. For this purpose, the use of geostatistical methods will be the best option, because in addition to estimating value of parameter in each point of desired space with the smallest possible error, the amount of uncertainty or estimation error can also be obtained. Usually estimation results will be accompanied with evenness (Deutsch, Wen 2000).

Access to Asmari Formation during drilling is of great importance. In this study, a model was presented using geostatistical methods and kriging estimator that helps predict top of Asmari Formation with very high accuracy before drilling operations.

Predicting the top of formation before drilling plays a key role in well drilling planning.

Geostatistical modelling of the top of Asmari formation by kriging is highly accurate in estimating unknown points, as in the tests for evaluating accuracy and precision of the estimated model and the error distribution; the kriging method shows a very good function. To evaluate the accuracy of this model, location of the three drilled wells in which the top of Asmari formation value was known was compared with the designed model. This survey shows that the value of estimated parameter is very close to the true value (Table 3 ).

Table 3. Comparison real value and estimated value by the model

\begin{tabular}{c|c|c}
\hline Well Number & Real Value & Estimated Value \\
\hline Well 1 & -3515 & -3514 \\
\hline Well 2 & -3687 & -3686.5 \\
\hline Well 3 & -3493 & -3494 \\
\hline
\end{tabular}

\section{References}

Christakos, G. 2001. Modern Spatiotemporal Geostatistics. Oxford University Press. 312 p. ISBN 0195138953.
Corstange, R.; Grunwald, S.; Lark, R. M. 2008. Inferences from fluctuations in the Local Variogram about the assumption of stationary in the variance, Geoderma 143: 123-132. http://dx.doi.org/10.1016/j.geoderma.2007.10.021

David, M.; Blais, R. A. 1977. Geostatistical Ore Reserve Estimation, Developments in Geomathematics 2: 27-33.

Deutsch, C. V. 2002. Geostatistical Reservoir Modelling. Oxford University Press, Oxford. 376 p. http://dx.doi.org/10.1023/A:1007502817679

Deutsch, C. V.; Wen, X. H. 2000. Integrating large-scale soft data by simulated annealing and probability constraints, Math. Geol. 32(1): 49-67.

Dimitrakopoulos, R.; Desbarats, A. J. 1997. Geostatisticalmodeling of grid block permeabilities for 3D reservoir simulators, SPE Reservoir Engineering 8: 13-18.

Gotway, C. A.; Young, L. J. 2002. Combining incompatible spatial data, Journal of the American Statistical Association 97: 632-648. http://dx.doi.org/10.1198/016214502760047140

Haining, R. 2003. Spatial Data Analysis: Theory and Practice. Cambridge: Cambridge University Press. http://dx.doi.org/10.1017/CBO9780511754944

Hansen, T. M.; Journel, A. G.; Tarantola, A.; Mosegaard, K. 2006. Linear inverse Gaussian theory and geostatistics, Geophysics 71(6): 101-111.

Kelsall, J.; Wakefield, J. 2002. Modeling spatial variation in disease risk: a geostatistical approach, Journal of the American Statistical Association 97: 692-701. http://dx.doi.org/10.1198/016214502388618438

Ortiz, C. J.; Deutsch, C. V. 2002. Calculation of uncertainty in the variogram, Math. Geol. 34(2): 169-183. http://dx.doi.org/10.1023/A:1014412218427

Webster, R.; Oliver, M. A. 2007. Geostatistics for Environmental Scientists. Statistics in Practice, Wiley, Chichester. http://dx.doi.org/10.1002/9780470517277

Mohammad ABDIDEH. PhD in Geology, Assistant Professor. Department of Petroleum Engineering, Omidiyeh Branch, Islamic Azad University, Omidiyeh, Iran. Ph +98 611444 816, e-mail: m.abdideh@yahoo.com. Research interests: petroleum geology, geostatistical modeling, petrophysics, rock mechanics, geophysics.

Majed ABYAT. Msc in Petroleum Engineering. Department of Petroleum Engineering, Omidiyeh Branch, Islamic Azad University, Omidiyeh, Iran. Research interests: petroleum engineering, geostatistical modeling, petrophysics. 